\title{
The effects of training on passive avoidance deficits in rats with hippocampal lesions:' A reply to Isaacson, Olton, Bauer, and Swart
}

\author{
DONALD G. STEIN ${ }^{2}$ AND ROBERT J. KIRKBY \\ UNIVERSITY OF OREGON
}

Animals with bilateral cortical or hippocampal lesions were given either 5 or 10 days of approach training and tested for passive avoidance of shock. Passive avoidance was impaired in long-trained hippocampal rats whereas shorttrained counterparts did not differ from cortical controls. Cortically lesioned rats were not affected by amount of pretraining. Results indicate that amount of prior training does effect passive ability in animals with hippocampal damage.

In a recent article, Kimble, Kirkby, \& Stein (1966) examined the behavior of rats with hippocampal lesions in two types of passive avoidance task. The first situation required the inhibition of a "spontaneous" step through response and produced no deficit in performance in comparison with cortically lesioned animals. The second task required the inhibition of a previously trained approach response, and in this situation, the animals with hippocampal lesions were unable to demonstrate passive avoidance of shock. The authors suggested that the deficit produced by hippocampal damage could be explained in terms of response perseveration resulting from the prior training.

The hypothesis that prior training conditions could, in part, influence passive avoidance behavior in animals with hippocampal lesions was recently questioned by Isaacson, Olton, Bauer, \& Swart (1966). Using latency as a measure of passive avoidance performance, they reported a "partial confirmation" of the Kimble, Kirkby and Stein results, but then claimed that (since) "impaired passive avoidance can be exhibited without specific, prior training indicates that it is not the training per se which is essential to the passive avoidance deficiency ..."

The present experiment was designed to study the effects of different amounts of prior training on passive avoidance learning in animals with hippocampal lesions. On the basis of the results of our initial study, we predicted that long pre-training would inhibit passive avoidance in animals with hippocampal lesions, while a short period of pre-training would have less of an effect.

\section{Procedure}

The apparatus used in the present experiment has been described in detail elsewhere (Kimble, 1963; Kimble, Kirkby, \& Stein, 1966). Twenty-eight male albinc rats, approximately 160 days old served as Ss. All of the animals were used in a previous maze learning study (Stein \& Kimble, 1966). Lesions were made at 80 days of age in one stage under pentobarbital anesthesia. Fourteen rats received bilateral damage to the hippocampus and 14 animals were subjected to bilateral aspiration of cortex directly overlying the hippocampus. Detailed histology has been reported elsewhere (Stein, 1965; Stein \& Kimble, 1966).$^{3}$

The Ss were placed on a 23-hr. 50-min. water deprivation schedule but were permitted access to food at all times. This schedule was begun two days prior to training and maintained for the duration of the experiment.

Seven cortically lesioned animals and seven with lesions in the hippocampus were given five days of training (six trials per day) during which they were permitted to leave the start box, traverse the length of the alley, and drink for a few seconds. The same procedure was carried out for the remaining 14 animals except that they received 10 days of training with six trials per day.

Testing for perseveration of the approach response was carried out for two days immediately following the training period. On the test days, the metal water spout was electrified, so that if the animal touched the spout, it received approximately a 2 ma shock to the mouth.

On the first day of testing, each S received two trials as usual; however, on the third trial the rats received shock upon touching the spout. The number of shocks received during a 15-sec. interval was used as the criterion for perseveration. The same criterion was used on the fourth and last trial. This procedure was repeated for the second day of avoidance training. Results

An analysis of variance indicated that length of training period and type of lesion had an effect on passive avoidance performance. In general, the animals with hippocampal damage tended to suffer more shocks than the cortical controls. In addition, the long period of training showed a tendency to influence passive avoidance performance in both experimental groups, (see Table 1).

The findings relevant to our hypothesis were obtained by individual comparisons between the groups. Hippocampal animals given long training (10 days) received significantly more shocks $(p<.01, F=9.15, d f=1 / 24)$ than their short-trained (5 days) counterparts, and also showed significantly more perseveration $(p<.05$, 
Table 1.

Total number of shocks received following periods of either Iong ( 10 days) or short ( 5 days) approach training in a straight alley. $N=7$ in each experimental group.

\begin{tabular}{lccc} 
& $\begin{array}{c}\text { Hippocampal } \\
\text { Lesions }\end{array}$ & $\begin{array}{c}\text { Cortical } \\
\text { Lesions }\end{array}$ & Totals \\
\hline Long-Training & 21 & 12 & 33 \\
Short-Training & 11 & $\frac{7}{19}$ & 18 \\
Totals & 32 & 19 & 51 \\
\hline
\end{tabular}

$F=7.42, d f=1 / 24$ ) than the long-trained cortically lesioned rats. In contrast, the short-trained animals with hippocampal lesions did not differ significantly from either the cortically lesioned short- or long-trained groups. Finally, no significant differences were obtained between the two cortically damaged groups. Discussion

We found that prior training does, in fact, affect the degree of the passive avoidance deficiency found in animals with bilateral hippocampal lesions. Isaacson, Olton, Bauer and Swart used latency measures as their dependent variable and reported differences between cortical and hippocampal animals regardless of the number of training trials given; although the deficit did increase with an increase in the number of trials. In the Isaacson experiment, the authors "utilized a response which would easily be performed by animals with or without training." The present experiment and the work by Kimble, Kirkby, and Stein both required a formal training period, and the response measure was not time-dependent. Differences in experimental design, apparatus and differences in the

\section{Comment}

\section{by R. L. Isaacson}

In our recent report (Isaacson, Olton, Bauer, \& Swart, 1966) we did not mean to imply that training was not an important variable in determining whether or not performance deficits will be exhibited by hippocampectomized animals. In fact, we had anticipated a rather strong training effect in the experiment. However, the results of the experiment, as well as of several pilot studies, led us to conclude that specific training may not be required. We proposed that training could be one variable which enhances a "total behavioral tendency" to make the response. Other things might also contribute to this tendency. I do not believe the results of the Stein and Kirkby experiment contradict this view. An interesting extension of their design would be to run subjects with both amounts of training under enhanced deprivation conditions which nature of the dependent variable may account for the differences in results. On the basis of the present study, however, we must agree with Snyder \& Isaacson (1965) that "the amount of specific training in the experimental situation should not be ignored as a distinguishing characteristic between the two passive avoidance problems." (p. 1289). We might also add that the amount of specific training should not be ignored within a particular passive avoidance problem.

\section{References}

Isaacson, R. L., Olton, D. S., Bauer, B., \& Swart, P. The effect of training trials on passive avoidance deficits in the hippocampectomized rat. Psychon. Sci., 1966, 5, 419-420.

Kimble, D. P., Kirkby, R. J., \& Stein, D. G. Response perseveration interpretation of passive avoidance deficits in hippocampectomized rats. J. comp. physiol. Psychol., 1966, 61, 141-143.

Kimble, D. P. The effects of bilateral hippocampal lesions in rats. J. comp. physiol. Psychol., 1963, 56, 273-283.

Snyder, D. R., \& Isaacson, R. L. Effects of large and small lesions on two types of passive avoidance responses. Psychol. Rep., 1965, 16, 1277-1290.

Stein, D. G. The effects of posttrial strychnine administration and hippocampectomy on learning and memory in the rat. Unpublished doctoral dissertation, University of Oregon, 1965.

Stein, D. G., \& Kimble, D. P. The effects of hippocampal lesions and posttrial strychnine administration on maze behavior $\hat{i}_{1,}$ the rat. J. comp. physiol. Psychol., 1966, in press.

\section{Notes}

1. Portions of this research were supported by a USPHS Predoctoral Research Fellowship to the first author and by USPHS Research Grant MH-08545-03 to Daniel P. Kimble.

2. Present address: Department of Psychology, Clark University. 3. A figure showing maximum and minimum extent of lesions may be obtained, upon request, from the first author. should increase the "total behavioral tendency" of the licking response. I would expect the hippocampectomized animals to make relatively more responses under these circumstances.

One point is of some theoretical concern in an experiment like that of Stein and Kirkby. Training was given in a specific runway type task, but the response measured was licking from a water bottle. Surely the licking response was highly overlearned before the experiment and it is difficult to see why a few trials more or less should have much effect upon it. From the paper it is not possible to determine whether the animals' tendencies to approach the area of the water spout was altered by the training or only the licking itself. The approach response is likely to be influenced by training. If it was not, one might consider investigating variables relating to habituation or distraction and their relation to the obtained results. 